"This is an Accepted Manuscript of an article published by Elsevier in Optical Switching and Networking on 2014, available online: http://dx.doi.org/10.1016/j.osn.2014.05.004. (C)2014. This manuscript version is made available under the CC-BY-NC-ND 4.0 license http://creativecommons.org/licenses/by-nc-nd/4.0/

\title{
Measuring the Survivability of Networks to Geographic Correlated Failures
}

\author{
Xuelian Long ${ }^{\mathrm{a}}$, David Tipper ${ }^{\mathrm{a}}$, Teresa Gomes ${ }^{\mathrm{b}}$ \\ ${ }^{a}$ Graduate Telecommunications and Networking Program \\ University of Pittsburgh

\begin{abstract}
Wide area backbone communication networks are subject to a variety of hazards that can result in network component failures. Hazards such as power failures and storms can lead to geographical correlated failures. Recently there has been increasing interest in determining the ability of networks to survive geographic correlated failures and a number of measures to quantify the effects of failures have appeared in the literature. This paper proposes a the use of weighted spectrum to evaluate network survivability regarding geographic correlated failures. Further we conduct a comparative analysis by finding the most vulnerable geographic cuts or nodes in the network though solving an optimization problem to determine the cut with the largest impact for a number of measures in the literature as well as weighted spectrum. Numerical results on several sample network topologies show that the worst-case geographic cuts depend on the measure used in an unweighted or weighted graph. The proposed weighted spectrum measure is shown to be more versatile than other measures in both unweighted and weighted graphs.
\end{abstract}

Keywords: Network survivability, geographic correlated vulnerabilities, weighted spectrum

\section{Introduction}

Communication networks are one of the critical national infrastructures upon which society depends $[1,2]$. Hence it is imperative that communication networks be designed to adequately respond to failures and attacks. This has led to significant interest in the design and analysis of communication networks, which are able to survive failures. In particular, the survivability of WDM optical technology based backbone networks are of concern due to the high volume of traffic carried.

Network survivability originated from a military viewpoint and is defined as the property of a system, subsystem, equipment, process, or procedure that provides a defined degree of assurance that the named entity will continue to function during and after a natural or man-made disturbance [3, 4, 5]. A communication network disturbance or failure is usually defined as a situation where the network is unable to deliver communication services. Thus a failure can be viewed as a disruption of service rather than degradation due to congestion. Typical failure events include cable cuts, hardware malfunctions, software errors, power outages, natural disasters (e.g., flood, fire, earthquake, etc.), accidents, human errors (e.g., incorrect maintenance) and malicious physical/electronic attacks. Traditionally, network survivability analysis has focused on the effects of random failures. However, many real-world causes of failures/attacks happen at specific geographic locations (e.g. power outage, floods, Electromagnetic Pulse attack [6], etc.), therefore, the effect of such failures/attacks is geographically correlated.

Only recently has work emerged from the critical infrastructure protection and network science communities studying the effects of correlated failures due to targeted attacks or geographically localized events (e.g., blackout, earthquake, etc.). This work typically models the network by a geographic coordinate based topological graph consisting of nodes and links. In the context of optical based wide area networks, the nodes are WDM access nodes or optical cross connects and links represent optical transmission systems consisting of long haul fiber and optical 
amplifiers. One of the main themes of the current literature, is finding the geographic locations of disasters or attacks that will have the largest impact on the survivability of a network. Specifically the focus is on determining which geographic location for a specific failure scenario (e.g., node failure [20, 2], geographic location of link cuts [13], geographic circular shaped failure area[18], etc.) has the greatest impact on the network. Alternatively this can be thought of as determining the weakest or most vulnerable portion of a network topology for a specific type of geographic based event. Unfortunately, how to measure the impact of a failure is an open issue and a variety of measures have appeared in the literature primarily focusing on the graph connectivity. A weakness of many of the currently adopted measures is requiring the network topology graph be unweighted. However, in many cases it is desirable to study weighted graphs where the weights represent network topology characteristics (e.g., link capacity, link availability, etc.). Additional, drawbacks of existing measures is requiring the network graph remain connected or alternately be partitioned after the failures. In general, the lack of a consistently used measures makes comparing and contrasting the existing literature difficult.

In this paper, we propose a new survivability measure applicable to both weighted and unweighted graphs. Further the proposed measure can be utilized to study connected and partitioned graphs. The measure is termed the weighted spectrum (WS) and is based on the eigenvalues of the normalized Laplacian of a graph. Fay et al., illustrate the theory and application of WS to Internet topology analysis in [12]. They use the distribution of Weighted Spectrum to examine the mixing properties of networks, and to derive optimal parameterizations of synthetic Internet topology generators, but do not mention it as a measure to evaluate the network survivability. Here, we propose using the variation of the WS in the presence of a geographic correlated failure as a performance measure (WS is formally defined in Section 3) to assess the network survivability. Given a network topology with geographic coordinates of the nodes, we use the algorithm proposed in [13] to obtain a set of potential vulnerable geographic linear cuts. We designate as the worst-case cut, according to a given performance measure $X$, the one which causes the greatest variation in $X$. Note, that more than one cut may result in the worst case variation in $X$. A comparative evaluation is conducted by contrasting the worst-case cut found by WS with the one(s) found by several other measures used in the literature. Additionally, we also find the worst-case node failure using WS and compare the result with other measures. We conduct numerical experiments on a variety of both unweighted and weighted real world optical backbone network topology graphs. The results show that the worst case cut depends on the measure adopted. Further, it is shown that the proposed Weighted Spectrum measure typically identifies a wider range of cuts of concern and can be used to study both weighted and unweighted network topology graphs for failure scenarios ranging from link/node failures where the topology is still connected to large scale disasters where the topology is partitioned.

The rest of the paper is organized as follows. We briefly discuss the related work in Section 2. In Section 3, we present the necessary theoretical background on the weighted spectrum, the other measures we compare against and the network survivability evaluation model. In Section 4, the results of our numerical experiments are presented including a comparison of the different measures. We conclude and discuss future research directions in Section 5.

\section{Related Work}

Spectral graph theory has been used to characterize the Internet topology. Fay et al. study the Weighted Spectral Distribution (WSD) to determine the level of underlying structural similarity between different graphs [12]. WSD can be used for topology tuning because of its low computational costs. It also can help investigate the evolution of the Internet graph and design future topology generators. However, [12] focuses on the graph comparison and does not mention anything about finding the vulnerable or critical part of the network.

Fiedler in [7], defined the algebraic connectivity of a graph as the second smallest eigenvalue of the Laplacian matrix determined from the topology graph. The larger the AC, the more difficult it is to cut a graph into disconnected components [7]. Jamakovic and Uhlig propose to use the AC to provide a measure of properties such as robustness of a network to failures in [14]. Liu et al. [15] studied the use of AC in evaluating survivable network designs. The network topologies considered were unweighted and not geographically coordinate based.

Tizghadam and Garcia $[9,8]$ propose the measure termed network criticality (NC) to analyze the robustness of a network to unexpected changes. The network criticality is determined from the trace of the inverse of the Laplacian matrix and can be related to the node and link betweenness. The smaller the NC, the less sensitive the network is to changes in its topology and traffic [9]. Bigdeli et al. [16] compare the properties of NC with different measures (e.g., 
AC) and propose guidelines for designing and simplifying complex networks based on the desired network properties. Note, that this work along with [9], did not study geographic coordinate based networks.

Neumayer et al., study the impact of geographical disasters on network robustness and survivability in [13, 17]. They focus on the problem of geographical network inhibition (i.e., finding the most vulnerable cut of geographic line segment or circle) in optical layer backbone networks. The measures studied include node degree (ND), total expected capacity (TEC) and the average two terminal reliability (ATTR). ND [10] is defined as the number of direct neighbors of node. The TEC is a form of node strength (NS) [11] which is the sum of weights of a node's connections in a weighted network. The average two terminal reliability is the fraction of node pairs that are connected by a path to each other.

Trajanovski, Kuipers and Van Mieghem [18] extend the approach of [17] to identify the critical regions of a network topology. A critical region is defined as that part of a topology that can be enclosed by an elementary figure (e.g., circle, rectangle, equilateral triangle, etc.) whose removal would result in the largest network disruption. They expand on the critical region analysis in [19] and define a region-disjoint path pair as two paths with minimum weights between a source destination pair that cannot both be failed by a circular regional cut. In both works, the average shortest path (ASP) and the number of disconnected pairs were used to estimate the level of network disruption.

Yan et al., [20] evaluate the criticality of assets in the Internet infrastructure at a national ISP and autonomous systems level. They use a large scale simulation based analysis to determine which set of node failures have the largest impact based on a graph-theoretical perspective, a route-based perspective, a traffic-based perspective or a consequence-based perspective.

Agarwal et al., [21] considers the effects of geographically correlated probabilitistic attacks. That is the probability of a network component failing varies with location and is geographically correlated. They provide algorithms to evaluate the effect of probabilistic failures in networks utilizing dedicated path protection for three measures: (1) the average number of components effected, (2) the average two terminal reliability and 3) the average maximum flow after failure.

Banerjee et al., [22] points out that most of the literature focuses on connectivity based measures many of which are ofthen not defined when the network topology is partitioned by a failure. They propose a metric termed region based component decomposition number (RBDCN) which measures the number of connected topology components after a failure. Additionally, they suggest that the partioned component networks be characterized by the smallest and largest component size.

\section{Measures and Model Studied}

We first present theoretical background material on the measures adopted in the literature and the weighted spectrum of a graph and then propose our evaluation model for determining the geographic cuts that have the largest impact on the network and thereby locate the most vulnerable part of the topology.

\subsection{Theoretical Background}

We represent the physical network topology as an undirected graph denoted by $G=(V, E)$, where $V$ is the set of vertices/nodes and $E$ is the set of edges/links. Let $M$ denote the number of network nodes, that is, the cardinality of the node set $(M=|V|)$. Each node $v \in V$, has two dimensional coordinates $\left(x_{v}, y_{v}\right)$ (e.g., latitude and longitude) and the spherical distance of a link connecting two nodes $u$ and $v$ is denoted by $\operatorname{Dis}(u, v)$. The adjacency matrix $A$ of a graph is defined as:

$$
a_{u, v}= \begin{cases}1 & \text { if }(u, v) \in E, \\ 0 & \text { otherwise. }\end{cases}
$$

The degree of a vertex/node $v$ in a unweighted graph is the number of neighbors of $v$ with which the node has a direct link. The degree of node $v$ can be determined by summing the associated row or column of the adjacency matrix. Many of the metrics adopted in the literature to evaluate topological vulnerabilities can be found directly from the basic topology graph description. For example, the average shortest path (ASP) is defined as the average over all shortest paths in the network between every $M \times(M-1)$ node pairs. The network diameter DIA is the longest shortest path between any of the node pairs in the network. Both the ASP and DIA can be determined from the topology graph by running any of a number of shortest path algorithms. 
As noted above, one is often interested in weighted graphs where the weights represent a characteristic of the network (e.g., link availability). A weighted undirected graph $G$ has weight function $w: V \times V \rightarrow \mathcal{R}$ satisfying

$$
w(u, v)=w(v, u)
$$

with $w(u, v) \geq 0$ and $w(u, v)=0$ if and only if $(u, v) \notin E(G)$. An unweighted graph is the special case where the weights are 0 or 1 . The degree of a vertex/node $v$ in a weighted graph (also termed the node strength) is defined as:

$$
d_{v}=\sum_{u} w(u, v)
$$

Let $D$ denote a diagonal matrix of weighted node degrees, and $L$ be the Laplacian matrix of the weighted graph. The Laplacian is defined as [23, 24]:

$$
L(u, v)= \begin{cases}d_{v} & \text { if } u=v \\ -w(u, v) & \text { if } u \text { and } v \text { are adjacent } \\ 0 & \text { otherwise }\end{cases}
$$

The normalized Laplacian matrix $\mathcal{L}$ associated with the weighted undirected graph as defined by

$$
\mathcal{L}=D^{-1 / 2} L D^{-1 / 2}
$$

or, equivalently:

$$
\mathcal{L}(u, v)= \begin{cases}1 & \text { if } u=v \text { and } d_{v} \neq 0, \\ -\frac{w(u, v)}{\sqrt{d_{u} d_{v}}} & \text { if } u \text { and } v \text { are adjacent, } \\ 0 & \text { otherwise. }\end{cases}
$$

A number of topology vulnerability assessment metrics can be determined from $L$ or $\mathcal{L}$. Let $\lambda$ denote the Laplacian spectrum of the graph, that is $\lambda$ is the set of eigenvalues of $\mathcal{L}$ found from $|\lambda I-\mathcal{L}|=0$. In the algebraic graph theory literature [24], it has been shown that zero is always an eigenvalue of the graph (i.e., $\lambda_{1}=0$ ), and the next smallest eigenvalue $\lambda_{2}$ is known as the algebraic connectivity (AC) of the graph. If the algebraic connectivity is zero (i.e., $\lambda_{2}=0$ ) then the network is partitioned. In fact, the number of zero eigenvalues [24] is equal to the number of connected components of the network which is an alternative method to determine the region based decomposition number (RBDCN) metric of [22]. The network criticality (NC) [9] is defined in terms of the Laplacian $L$ as

$$
N C=2 M \operatorname{Tr}\left(L^{+}\right)
$$

where $\operatorname{Tr}()$ is the trace of the matrix and $L^{+}$denotes the Moore- Penrose inverse of the Laplacian matrix L. In[9] it is shown that the $N C$ is related to the normalized weighted betweeness.

Here we propose the use of the weighted spectrum $W S$ as a metric and note that $W S$ can be related to $N$-cycles in a graph. An $N$-cycle in graph $G$ is defined in [12] to be a sequence of vertices $u_{1}, u_{2}, \ldots, u_{N}$ where $u_{i}$ is adjacent to $u_{i+1}$ for $i \in[1, N-1]$ and $u_{N}$ is adjacent to $u_{1}$. The Weighted Spectrum WS is given [12] as the normalized sum of $N$-cycles:

$$
W S(G, N)=\sum_{i}\left(1-\lambda_{i}\right)^{N}
$$

Given a graph $G$, it is not difficult to get its Normalized Laplacian Matrix and associated eigenvalues. Therefore the Weighted Spectrum can be determined if appropriate $N$ is chosen. Different values of $N$ indicate different graph properties. For example, the number of 3-cycles is the number of triangles in the graph, thus the Weighted Spectrum for $N=3$ is related to the weighted clustering coefficient. According to [12] the number of $N=4$ cycles is an important graph property related to the number of disjoint paths in a network and WS with $N=4$ is proportional to the number of disjoint paths. In the numerical results of the following sections we show the effects of varying $N$ on $W S$, but given the density of the considered test networks, primarily choose $N=4$ to calculate $W S$. 


\subsection{Evaluation Model for Vulnerable Cuts}

The network survivability evaluation model proposed here employs the results of an optimization problem to find the most vulnerable geographic locations. We make the same assumptions as [13] in that, there are no overlapping nodes on a plane in the geographic graph and that for collinear nodes the distance between the two farthest collinear nodes is larger than the length of the considered cut. We use the Geographical Network Inhibition by Line Segments (GNIL) model in [13] to determine a set of vulnerable geographic locations. Given a geo-spatial graph and the cut length $h$, GNIL is used to generate a set of cuts whose impact is evaluated for a given measure (e.g., AC). The worstcase cut or cuts (i.e., the cut may not be unique) is defined as the cut/cuts that will cause the largest change of the measure.

Here we propose the measure based on Weighted Spectrum to assess the impact caused by the geographic cuts. Given a graph, we use $W$ to denote the default WS of the original graph. Consider the geographic vulnerable cut $i$; we use $W_{i}^{\prime}$ to denote the WS of the changed graph where the link(s)/node(s) in cut $i$ have been removed. The difference between the WS of the changed graph and the default WS is taken as the objective function of the optimization problem, since the most vulnerable cut would cause the largest difference between the two WSs. Thus the optimization problem can be defined as:

$$
\max _{i}\left|W_{i}^{\prime}-W\right|
$$

where $|\cdot|$ indicates the absolute value function and $i$ represents the cuts in the set of identified vulnerable geographic cuts. In [13], it was shown that the GNIL algorithm creates a polynomial-sized subset of all possible cuts and the worst-case cuts are contained in the subset if the evaluation metric is monotonic. Note, that many of the measures in the literature are not monotonic (e.g. DIA) or the montonicity depends on the link weights (e.g., NC). Here we adopt the approach that for each measure (AC, NC, etc.), we first compute the measure for the topology graph, then determine the cut or cuts from GNIL that results in the largest change in the measure. These cut(s) are termed the worst-case cut(s) over the set evaluated. Since the cut(s) can result in isolated component graphs, we only consider the largest component of the original graph for evaluation of metrics that require connectivity (e.g., $A C, A S P, D I A$ ) whereas for other metrics (i.e., WS, NC), we still use the whole graph, even with isolated component(s).

The measures we study here are node degree (ND), average shortest path (ASP), network diameter (ND), algebraic connectivity (AC), node strength (NS), network criticality (NC) and weighted spectrum (WS) Note that measures such as ASP, ND, and DIA are typically defined for unweighted graphs and are function of the topology structure independent of the network geographic span (e.g. ASP $=3$ hops). Thus such measures give the same value regardless of weighted or unweighted graphs. Other measures such as NS, NC and WS can be applied to weighted or unweighted graphs.

Besides the aforementioned measures, we also tried to consider the average two terminal reliability ${ }^{1}$ (ATTR) measure for unweighed graphs used in [13] and region based decomposition number (RBDCN) [22]. However, as the cut length considered in this paper is between $15 \%$ and around $20 \%$ of the average geographic length of the links in the graph, ATTR and RBDCN are not sensitive to such cuts. This is because the real world topology graphs studied are all at least two connected and with a geographic span such that the network is not partitioned or the partition only isolates one node. This illustrates one of the advantages of WS metric in that is can assess small geographic correlated failures and as well as large failures that partition the network into many components.

\section{Numerical Results}

In this section we present numerical results to provide a comparative evaluation of a set of measures from the literature along with the proposed weighted spectrum measure. For simplicity, we use $A S P, D I A, W S, A C, N C, N D$ and $N S$ to denote the worst-case cut/cuts and nearly worst-case cuts determined by solving the optimization problem (9) with the corresponding measure. Specifically, for each measure, we first compute the measure for the original topology graph, then determine the cut or cuts that results in the largest change in the measure. This cut/cuts is termed the worst-case cut. If the ratio of the difference between the change caused by a non-worst-case cut and the change

\footnotetext{
${ }^{1}$ TTR between two nodes is 1 if there is a path between them, otherwise 0 [25].
} 
Table 1: Real-World Reference Networks[26]

\begin{tabular}{ccccccc}
\hline Number & Name & $M$ & $L$ & $h$ & $\bar{l}$ & $\overline{\bar{l}}$ \\
\hline 1 & Atlanta & 15 & 22 & 25 & 136.2806 & 0.1834 \\
2 & Abilene & 12 & 15 & 2 & 9.9839 & 0.2004 \\
3 & Cost266 & 37 & 57 & 1 & 4.9578 & 0.2016 \\
4 & Dfn-bwin & 10 & 45 & 0.6 & 3.5382 & 0.1696 \\
5 & pioro40 & 40 & 89 & 20 & 129.8363 & 0.1540 \\
6 & Germany50 & 50 & 88 & 0.2 & 1.1645 & 0.1724 \\
71 & Janos-US & 26 & 42 & 1 & 6.27 & 0.1595 \\
81 & Janos-US-CA & 39 & 61 & 1 & 5.50 & 0.1818 \\
9 & Nobel-EU & 28 & 41 & 0.8 & 4.6413 & 0.1724 \\
10 & Nobel-Germany & 17 & 26 & 0.3 & 1.7140 & 0.1754 \\
11 & Nobel-US & 14 & 21 & 2 & 11.8101 & 0.1693 \\
12 & Norway & 27 & 51 & 30 & 164.4903 & 0.1824 \\
13 & New York & 16 & 49 & 40 & 257.6191 & 0.1553 \\
14 & Pdh & 11 & 34 & 0.2 & 3.0286 & 0.1651 \\
15 & Polska & 12 & 18 & 0.4 & 2.3105 & 0.1732 \\
16 & France & 25 & 45 & 20 & 129.4876 & 0.1545 \\
\hline
\end{tabular}

caused by the worst-case cut over the changes caused by the worst-case cut is less than a threshold, then this nonworst-case cut is designated a nearly worst-case cut. This approach was followed in all sample networks, with the threshold for the nearly worst-case cuts $15 \%$ for $W S$ and $A C$, and $25 \%$ for $N C$ and $A S P$, respectively. We first consider a small graph as an example to show the results of our proposed measure and the aforementioned measures. This is followed by an exensive set of results for a group of real world optical network backbone topologies. All numerical results were obtained using MATLAB

\subsection{Example Case Study}

In this subsection, we will employ a small network as an example of using the proposed approach to evaluate geographic vulnerabilities. The network topology is shown in Figure 1 on a geographic grid. For a line cut value $h=10$, Figure 2 shows the worst-case cuts with the $A C$ as the evaluation measure. Figure 3 and Figure 4 show the worst-case cuts and nearly worst cuts with $N C$ and $A S P$ as the assessment measures, respectively. Figure 5 and Figure 6 show the worst-case cuts based on measures DIA and WS, respectively. From the figures one can see that the measures $A C, A S P, D I A$ and $W S$ find the same worst-case cuts for this topology. Measure NC finds different worstcase cuts compared with the other four measures but the nearly worst-case cuts found by $N C$ are the worst-case cuts found by other measures. Moreover, the nearly worst-case cuts found by measure ASP are the worst-case cuts based on the measure $N C$. From the results of this small topology, we can see that the different measures can actually find different worst-case cuts and nearly worst-case cuts (e.g., $N C$ and $A S P$ ). Since the example topology is small (6 nodes, 7 links), some measures (e.g., $A C, A S P, D I A$ and $W S$ ) result in the same worst-cast cuts. The worst-case cuts found by the majority of the measures disconnects the original topology into two disconnected components (one component with 2 nodes and the other with 3 nodes), thus these worst-case cuts have a large impact the network measures.

\subsection{Vulnerabilities of Realistic Topologies}

In order to better evaluate the metrics we chose 16 different real-world reference optical backbone networks from SNDLib [26] for a numerical study. The 16 networks are summarized in Table I, where $M$ is the number of network nodes, $L$ is the number of network links, $h$ is the geographic length of the cut in units corresponding to the network under study, and $\bar{l}$ is the average geographic length of links. Note, that $h$ is an input variable of GNIL and the value of the cut $h$ is adjustable to the geographic span of the network. Here, we choose the value of $h$ according to the average geographic length of the links in the graph (i.e., the ratio of $h$ over $\bar{l}$ is between $15 \%$ and around $20 \%$ for all 16 graphs). 


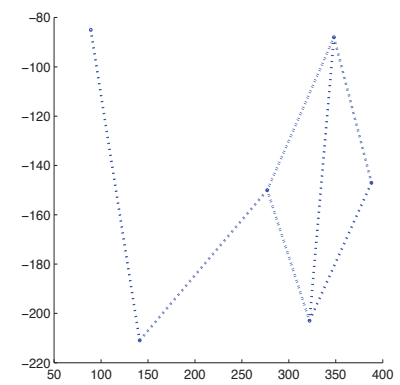

Figure 1: Topology of example network

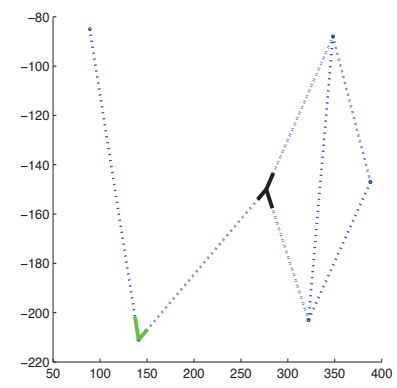

Figure 4: Cuts according to ASP for $\mathrm{h}=10$, black cuts are the worst-case cuts and green cuts are nearly worst-case cuts.

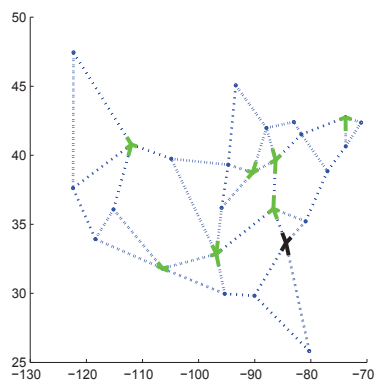

Figure 7: Cuts according to WS, black cuts are the worst-case cuts and green cuts are nearly worst-case cuts.

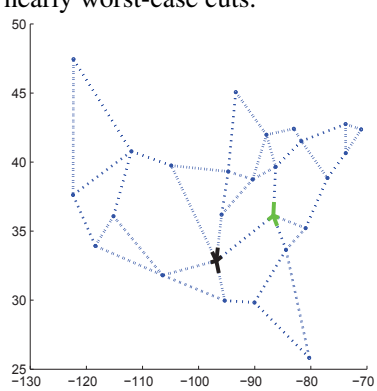

Figure 10: Cuts according to ASP, black cuts are the worst-case cuts and green cuts are nearly worst-case cuts.

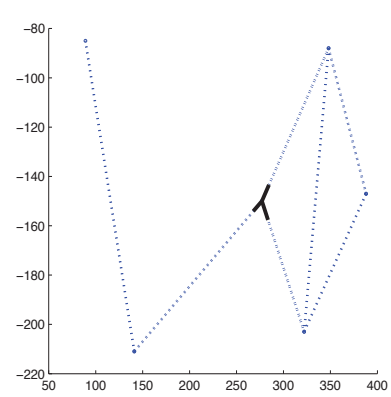

Figure 2: Cuts according to $A C$ for $\mathrm{h}=10$, black cuts are the worst-case cuts.

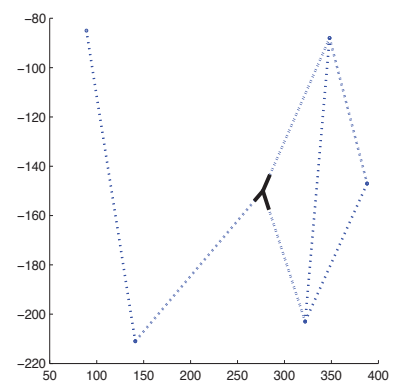

Figure 5: Cuts according to DIA for $\mathrm{h}=10$, black cuts are the worst-case cuts.

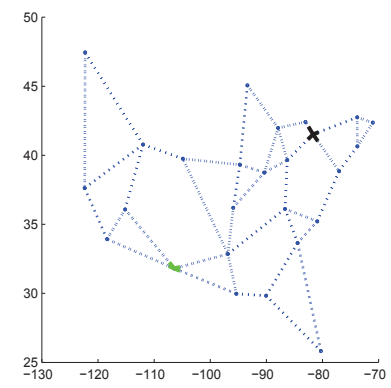

Figure 8: Cuts according to $A C$, black cuts are the worst-case cuts and green cuts are nearly worst-case cuts.

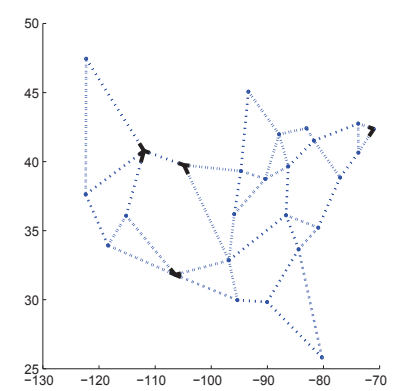

Figure 11: Cuts according to DIA, black cuts are the worst-case cuts.

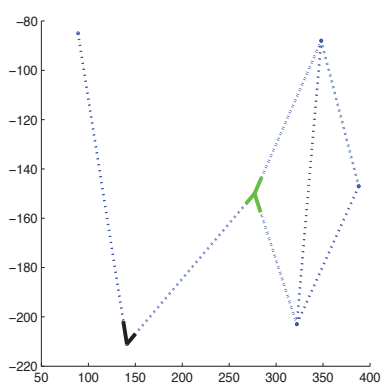

Figure 3: Cuts according to $N C$ for $\mathrm{h}=10$, black cuts are the worst-case cuts and green cuts are nearly worst-case cuts.

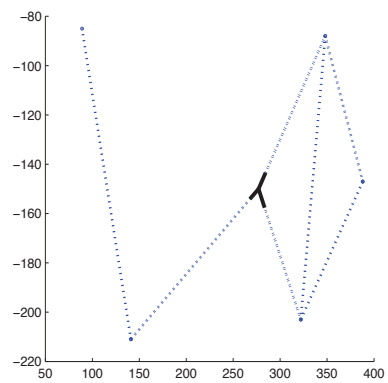

Figure 6: Cuts according to $W S$ for $\mathrm{h}=10$, black cuts are the worst-case cuts.

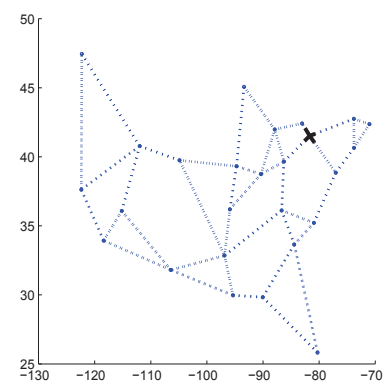

Figure 9: Cuts according to $N C$, black cuts are the worst-case cuts.

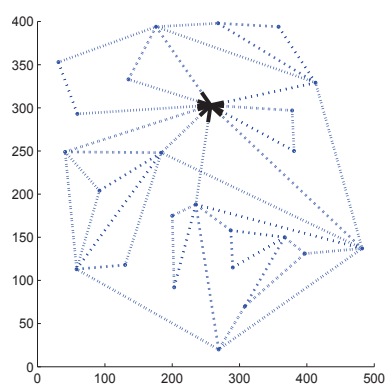

Figure 12: Cuts according to all measures, black cuts are the worst-case cuts. 


\subsubsection{Unweighted Graphs}

First we considered the effects of cuts on unweighted topology graphs for all 16 sample networks, with the results for network 7 (Janos-US) in Table I shown in Figures 7 to 11 for WS, AC, NC, ASP and DIA. We do not plot nearly worst-case cuts for DIA in Figure 11, since there are many such cuts. From these figures, we can see that for this network the worst-case geographic line cut according to $N C$ and $A C$ is the same. For the other three measures, the results are different. Note that for DIA, 13 worst-case cuts are identified. This is not surprising since the network diameter often will exist between several pairs of nodes. We observe, that the nearly worst-case cuts vary depending on the measure considered. Visual assessment of the worst-case and near worst-case cuts for other unweighted topology graphs show the same phenomenon and in some cases the worst-case cuts selected are exactly the same. Figure 12 shows such a case for network 16 (France).

In order to compare the measures in a quantifiable fashion, given a worst-case geographic line cut found by a certain measure we use all the other measures to evaluate this cut. For example, for the worst-case geographic line cut found by $A C$, we will use other measures (e.g. $W S, N C$, etc.) to evaluate the worst-case cut's impact on the network topology. If the worst-case geographic line cut found by a specific measure has an impact that is very close to the largest impact on all the other measures, it might be the best measure for evaluating the geographic vulnerabilities.

We define $r_{\mu, \eta}=\left|\frac{\mu\left(G_{\eta}^{\prime}\right)-\mu(G)}{\mu\left(G_{\mu}^{\prime}\right)-\mu(G)}\right|$ as the evaluation ratio, where $\mu(G)$ denotes the function of applying measure $\mu$ to graph $G$ and $G_{x}^{\prime}$ denotes the changed graph after removing the worst-case cut found by the measure $x$. Therefore $r_{\mu, \eta}$ evaluates the ratio of the variation in measure $\mu$ due to the worst-case cut found by measure $\eta$ over that using measure $\mu$ on the graph with the worst-case found by measure $\mu$. Since $\left|\mu\left(G_{\eta}^{\prime}\right)-\mu(G)\right|$ is always smaller than or equal to $\left|\mu\left(G_{\mu}^{\prime}\right)-\mu(G)\right|, r_{\mu, \eta}$ is between 0 and 1 . If the measure $\eta$ finds the same worst-case cut as that using measure $\mu, r_{\mu, \eta}$ gets the maximum value 1 .

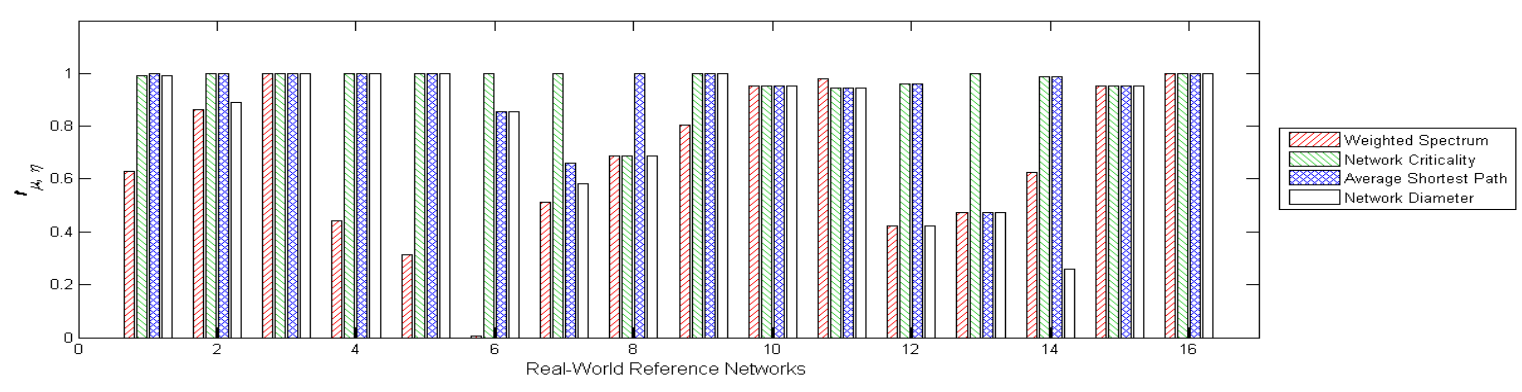

Figure 13: Evaluation by $A C$

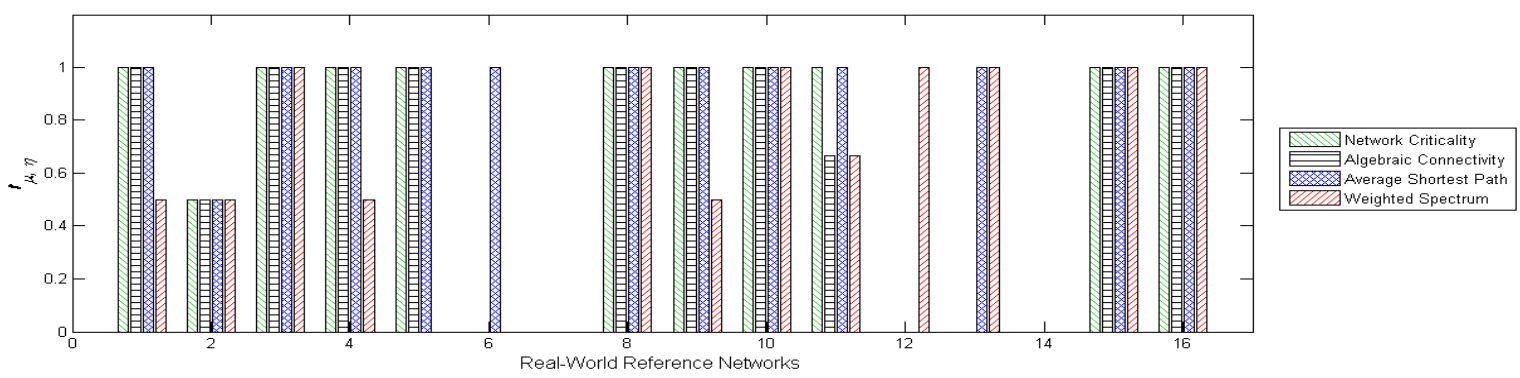

Figure 14: Evaluation by DIA

\footnotetext{
${ }^{1}$ In SNDlib these two networks are directed, however, we treat them as undirected graph in this paper. Therefore, the number of links are halved. Moreover, we use the average of the two directed links' costs between two nodes as the link cost in an undirected graph.
} 


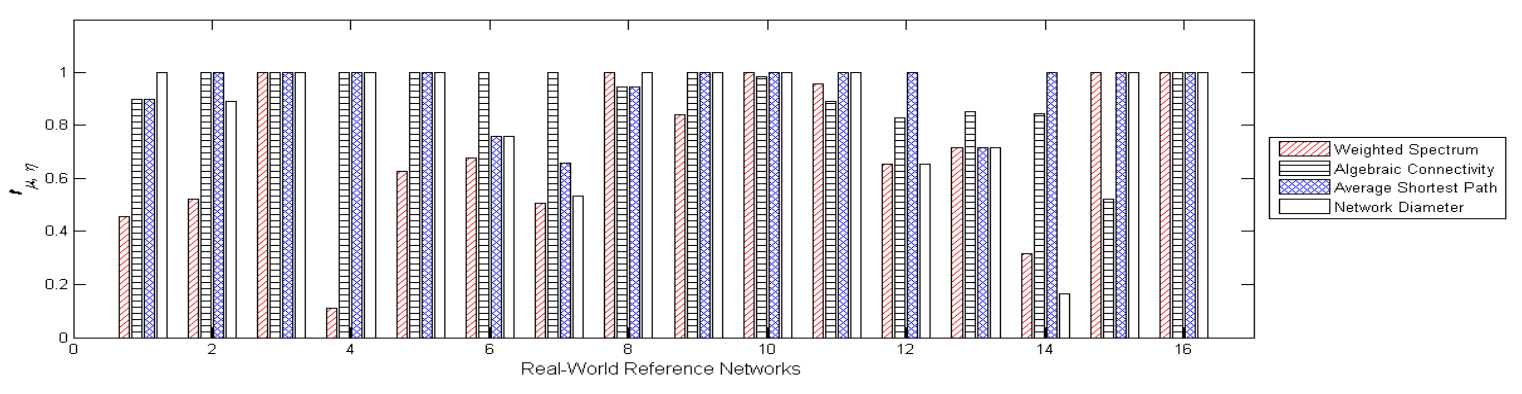

Figure 15: Evaluation by $N C$

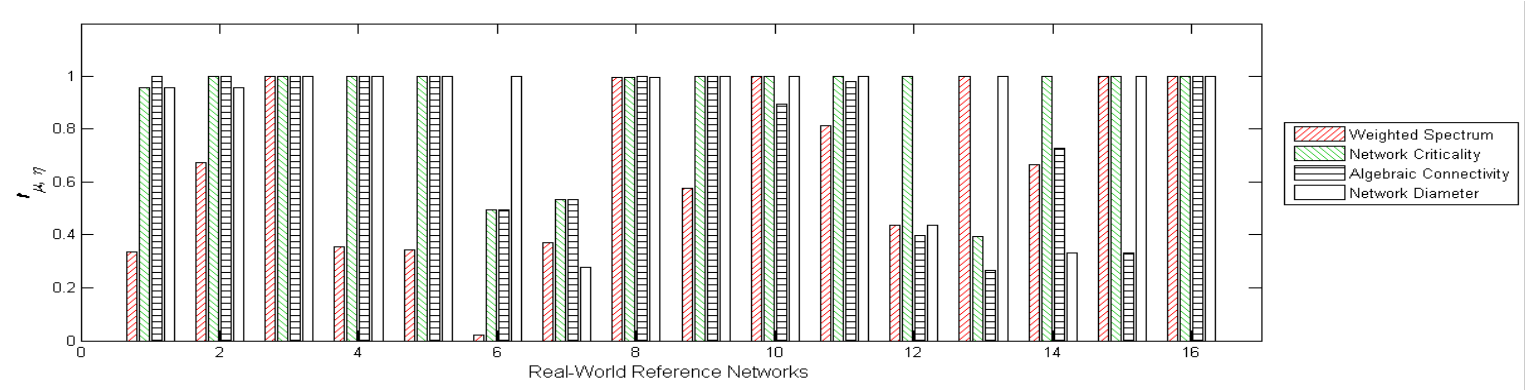

Figure 16: Evaluation by ASP

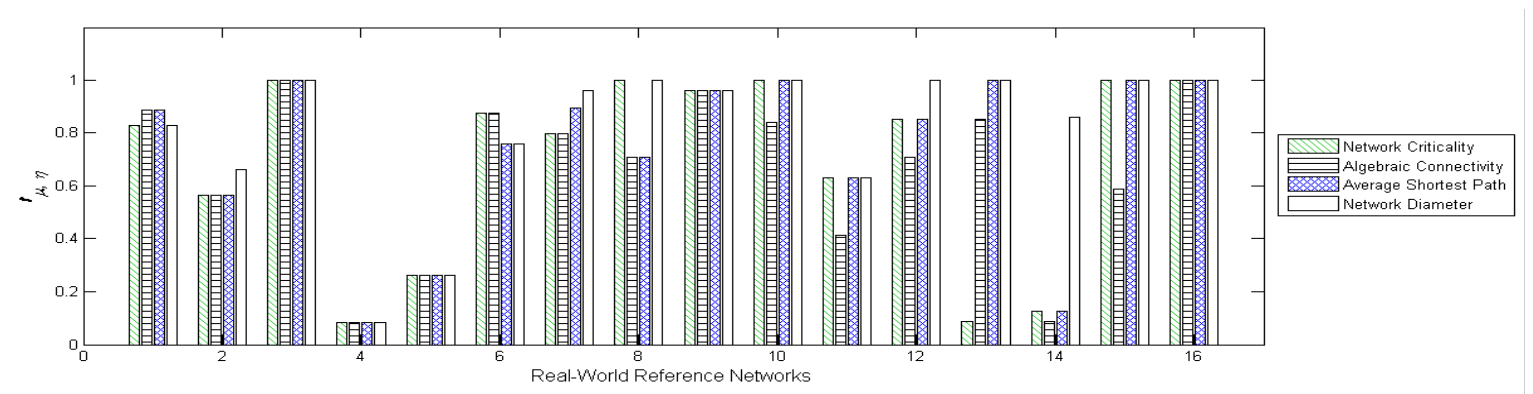

Figure 17: Evaluation by WS

The numerical results for the 16 sample networks considered are shown in figs. 13 to 17 for $A C, D I A, N C, A S P$ and WS respectively. In these figures, the $x$-axis represents the 16 different networks described in Table I. The $y$-axis denotes the evaluation measure $r_{\mu, \eta}$. Since DIA typically finds many worst-case cuts, if one coincides with the worstcase cuts determined by other measures it is selected for evaluation. From these five figures, we can observe that there is no one measure $\eta$ that can always achieve $r_{\mu, \eta}=1$ for all $\mu$ in all 16 networks. However, in many networks, several measures will find the same worst-case geographic line cut (i.e. $r_{\mu, \eta}=1$ ). From fig. 13 and fig. 15, observe that $A C$ and $N C$ give results that are close. Note, that the proposed performance measure WS typically identifies different cuts than the other measures, providing a somewhat unique measure.

\subsection{Vulnerable Line Cuts of Weighted Graphs}

One of the advantages of WS is the ability to study weighted graphs. In reality, network topologies will have variation in the cost, capacity and availability of the network links and nodes. Here we illustrate the use of WS for weighted topology graphs. Results are given for three networks (Janos-US, New York and Pioro40 - numbers 7 , 13 and 5, respectively, in Table 1) We consider two different link weights based on availability, energy consumption 
weights and another weight using the optical bandwidth module cost. The first two weights are defined based on the physical cable availability [27]:

$$
\begin{gathered}
W_{i, j}=\frac{-1}{\log _{2}\left(1-\frac{158 * \operatorname{Dis}(i, j)}{400 * 365 * 24 * 60}\right)} \\
W_{i, j}^{\star}=\frac{1}{W_{i, j}}=-\log _{2}\left(1-\frac{158 * \operatorname{Dis}(i, j)}{400 * 365 * 24 * 60}\right)
\end{gathered}
$$

where $\operatorname{Dis}(i, j)$ is the physical link distance between node $i$ and node $j$. In the case of eq. (10), the links with high availability have larger weights. On the other hand, in the second case of eq. (11), the links with high availability have lower weights. The energy consumption [28] weights are defined as:

$$
E_{i, j}=P C_{i, j} *(\operatorname{Dis}(i, j) / 80)+2
$$

where $P C_{i, j}$ is the power consumption as a function of link capacity. We assume the data rate of all links is $80 \mathrm{~Gb} / \mathrm{s}$ and the energy consumption for such $80 \mathrm{~Gb} / \mathrm{s}$ link is $P C_{i j}=9$ Watts. The optical bandwidth module cost weight is available from [26] for each link in the topologies considered.

Figures 18(a) to 18(d) show the worst-case cuts according to WS based on different link weights in the JanosUS network using $W_{i, j}, W_{i, j}^{\star}$, energy consumption and the module cost from the left to right sides, respectively. Figures 19(a) to 19(d) show the same results according to the $N C$ measure. Figures 20(a) to 20(d) and Figures 21(a) to 21(d) give the corresponding WS and NC results for the New York network. Similarly, Figures 22(a) to 22(d) and Figures 23(a) to 23(d) illustrate the cuts according to WS and $N C$ for the Pioro40 network. From Figure 18(a) and Figure 19(a) we can see that the worst-case cuts according to $N C$ are in the nearly worst-case cuts set according to WS. However, $N C$ does not consider the worst-case cuts determined by WS as the most vulnerable ones in the weighted graph defined with $W_{i, j}$. In Figure 19(b) and Figure 19(c), there is no nearly worst-case cuts addressed by $N C$. Comparing the result for the more dense New York network given in Figure 20 and Figure 21 one can see similar behavior with NC and WS identifying different worst-case cuts for the availability based and energy consumption measures, but in this case they are in agreement on the worst-case cut for the module cost weights. Moreover, in the Pioro40 topology, the worst-case cuts found by WS for the two availability based weights and energy consumption are nearly worst-case cuts found by NC, which indicate that WS could be a good measure as the worst-case cuts found by it can also cause large impacts on the NC measure.

\subsection{Vulnerable Nodes of Graphs}

Nodal Degree (ND) in unweighted graphs and Node Strength (NS) in weighted graphs are usually taken as important evaluation measures. The larger the ND/NS of a node, the more vulnerable the node would be, since more connections/more important connections would be lost once a failure happens at this node. However, ND/NS is not a macroscopic evaluation measure which can characterize the overall network connections. Here, we show the most vulnerable node found by ND and WS in an unweighted graph, as well as that according to NS and WS in a weighted graph. We still use the Janos-US topology in this experiment. The most vulnerable node found in an unweighted graph is shown in Figures 24(a) and 24(b) according to $N D$ and WS, respectively. The results for weighted graphs are shown in Figures 25 to 27.

We can see that in the unweighted graph, both $N D$ and $W S$ choose the node with 5 links as the most vulnerable node. In all three weighted graphs, $N S$ and $W S$ also find the same node as the most vulnerable node. Thus $W S$ is helpful in evaluating crucial nodes in both unweighted graphs and weighted graphs.

\subsection{Further analysis}

Considering that WS and NC capture different but relevant network vulnerabilites, the identification of worst case cuts using these two measures coud benefit from a bi-criteria analysis. In multiple-criteria optimization problems, the notion of an optimal solution (usually infeasible) is replaced by the concept of non-dominated solutions. A nondominated solution is a feasible solution such that no improvement in any criterion is possible without sacrificing at least one of the other criteria. 


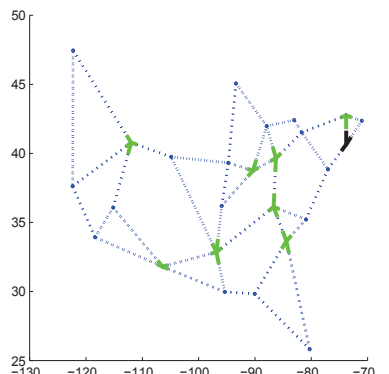

(a) $W_{i, j}$

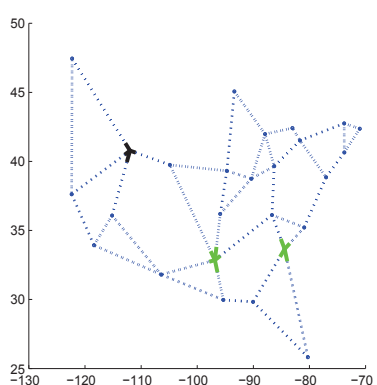

(b) $W_{i, j}^{\star}$

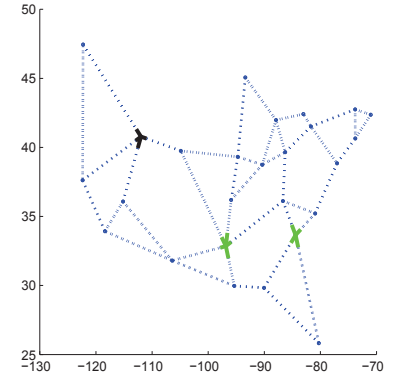

(c) energy consumption

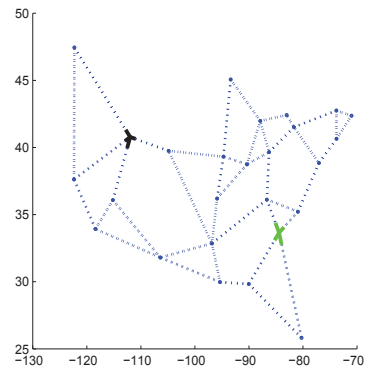

(d) module cost

Figure 18: Worst-case cuts and Nearly Worst-case cuts of the weighted Janos-US graph by WS

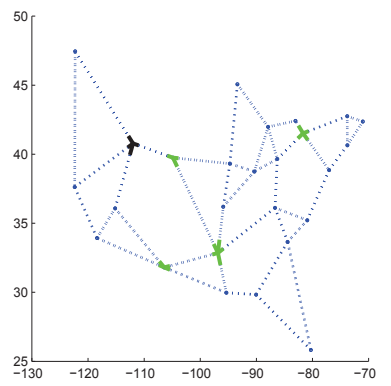

(a) $W_{i, j}$

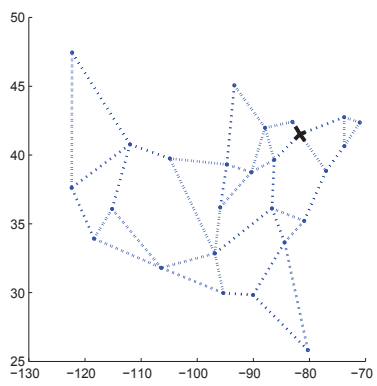

(b) $W_{i, j}^{\star}$

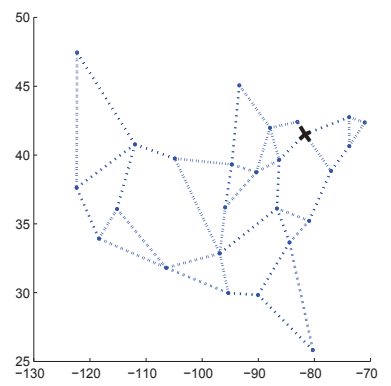

(c) energy consumption

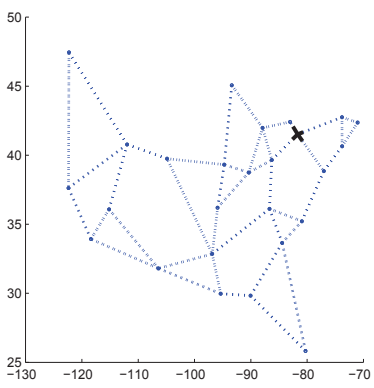

(d) module cost

Figure 19: Worst-case cuts and Nearly Worst-case cuts of the weighted Janos-US graph by NC

In this subsection, we employ a 2-dimensional figure to investigate the impacts of the potential cuts to two different measures WS and NC in weighted graphs. Moreover, we also would like to study the behavior of WS with different values fo the parameter $N$. We choose the Janos-US topology and run the experiments for the four different weights of 4.3 and five different values of parameter $N$. The results are shown in Figures 29(a) to 29(d).

For a point $(x, y)$ in the figure, $x$ is the ratio of the change of $N C(\Delta N C)$ to the maximum change of $N C(\max (\Delta N C)$; $y$ is the ratio of the change of $W S(\Delta W S)$ of out maximum change of $W S(\max (\Delta W S)$. For $x$ and $y$ the maximum change is caused by the worst-case cuts of $N C$ and $W S$ respectively. $N o d e(1,1)$ in the figure indicates the two different measures find the same worst-case cuts. The horizontal dash lines are reference lines which indicate the impacts of the worst-case cuts found by $N C$ to the WS. The vertical dash lines are reference lines which indicate the impacts of the worst-case cuts found by WS to the $N C$ measure. From Figure 29(a) we can see that WS and NC do not find the same worst-cast cuts. However, for the other three weights function (e.g., Figures 29(b) to 29(d)), the WS and NC can find the same worst-case cuts when $N=5$. Generally, the closer to $\operatorname{Node}(1,1)$, the cuts can achieve nearer worst-case performances based on both measures.

In Figure 29(a) we can see that for $N=4$ that $\mathrm{NC}$ does not consider relevant the worst case cut according to WS - the corresponding point has $\Delta N C /(\max \Delta N C)$ less than 0.2 . On the other hand we have a non-dominated solution with $\Delta N C /(\max \Delta N C)$ equal to 0.9 and just below 1 for $\Delta W S /(\max \Delta W S)$.

For $N=4$ let $\left(x_{1}, 1\right)$ and $\left(1, y_{1}\right)$ be the values corresponding to the points where $\Delta N C /(\max \Delta N C)$ and $(\triangle N C /(\max \Delta N C), 1)$ take the value 1 , respectively. In Figure 29(c) for $N=4$ we can see several points in the rectangle defined by $\Delta N C /(\max \Delta N C)>x_{1}$ and $\Delta W S /(\max \Delta W S)>y_{1}$, and at least one of them is a non-dominated solution considering the maximization of both $\triangle N C /(\max \Delta N C)$ and $\triangle N C /(\max \Delta N C)$. Considering that $\mathrm{NC}$ and WS are both important measures for evaluating network survivability regarding geographic correlated failures, identifying the cuts that correspond to non-dominated solutions (and possibly those in their close proximity) according to those two measures, may prove to be a good approach and requires further study. 


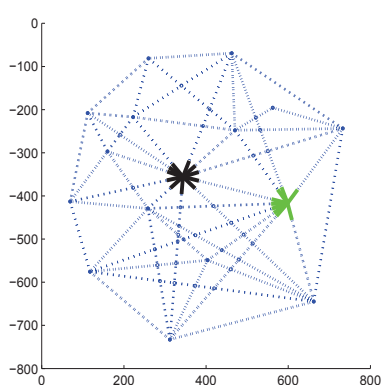

(a) $W_{i, j}$

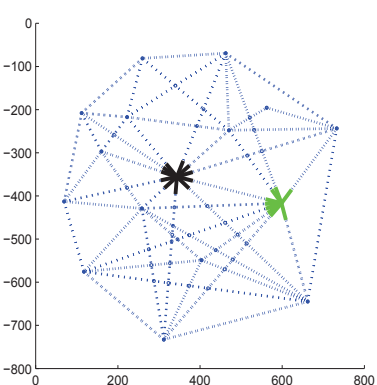

(b) $W_{i, j}^{\star}$

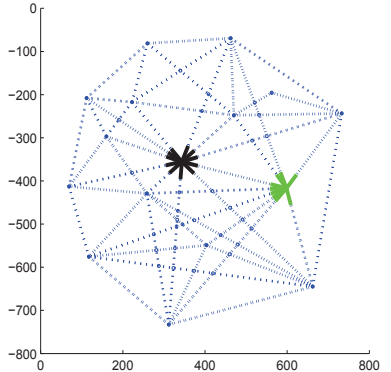

(c) energy consumption

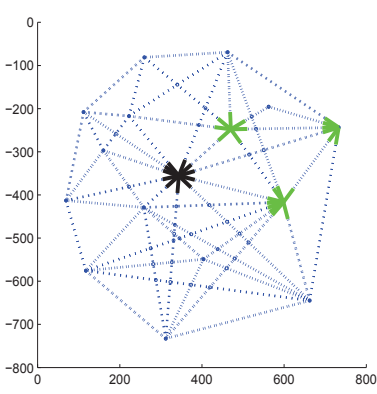

(d) module cost

Figure 20: Worst-case cuts and Nearly Worst-case cuts of the weighted New York graph by WS

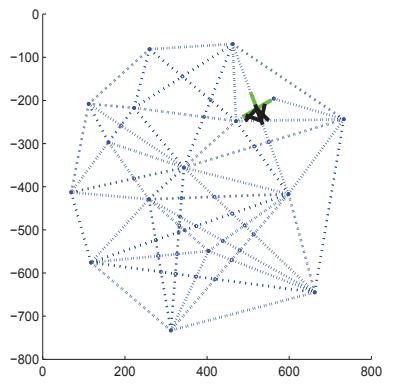

(a) $W_{i, j}$

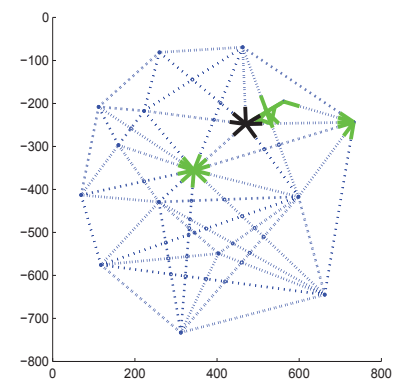

(b) $W_{i, j}^{\star}$

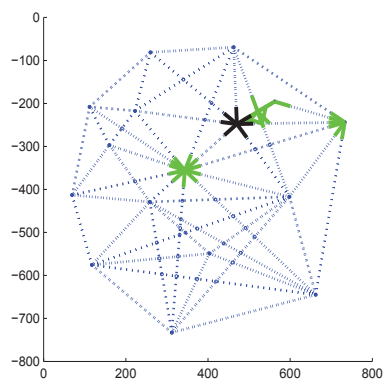

(c) energy consumption

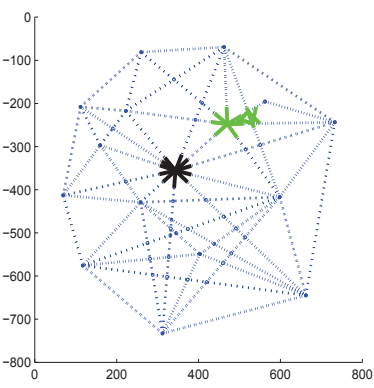

(d) module cost

Figure 21: Worst-case cuts and Nearly Worst-case cuts of the weighted New York graph by NC

\section{Conclusion}

In this paper, we proposed using the maximum variation on the Weighted Spectrum (WS) to measure the survivability of networks to geographic correlated failures. Through solving an optimization problem on WS, we can find the most vulnerable geographic locations. Numerical results using realistic network topologies were given comparing the worst-case cut obtained by WS with the results obtained using other common survivability measures in the literature (i.e., Algebraic Connectivity, Network Criticality, Average Shortest Path, Network Diameter). The numerical results show that in general the measures are not consistent in selecting a most vulnerable portion of the network and that $W S$ is the least correlated with the other metrics. Unlike most of the measures WS can also deal with weighted graphs and it was shown to have differing results than Network Criticality. Furthermore, with regard to finding the most vulnerable node, WS provides results consistent with the existing measures of Node Degree (ND) and Node Strength $(N S)$ in unweighted and weighted graphs respectively. From all the experiments we can conclude that WS is the most versatile measure in that it can be used to evaluate geographically correlated vulnerable links and vulnerable nodes in an unweighted graph and a weighted graph. Future work includes investigating WS under different failure scenarios (e.g., circular area shaped failures).

\section{Acknowledgement}

The work of Teresa Gomes has been partially supported by FCT under project grant PEst-C/EEI/UI0308/2011, and by Project CENTRO-07-0224-FEDER-002003. The work of David Tipper was supported in part by the US Army Research Office under the Multi-University Research Initiative (MURI) grant W911NF-07-1-0318. 


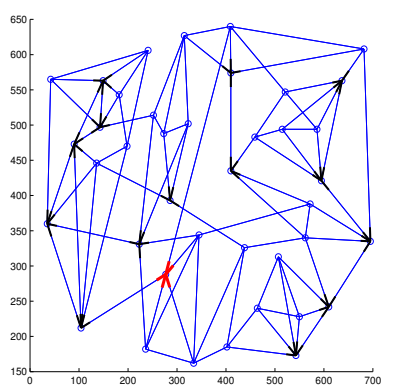

(a) $W_{i, j}$

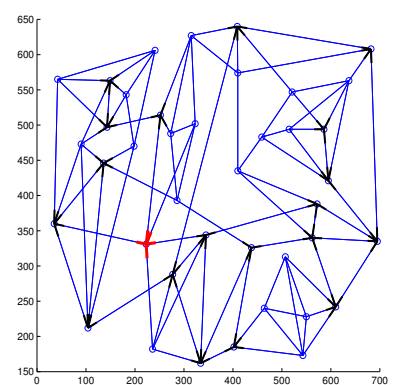

(b) $W_{i, j}^{\star}$

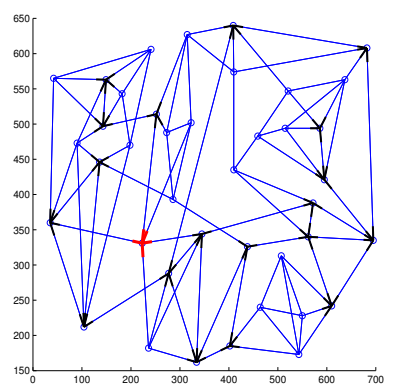

(c) energy consumption

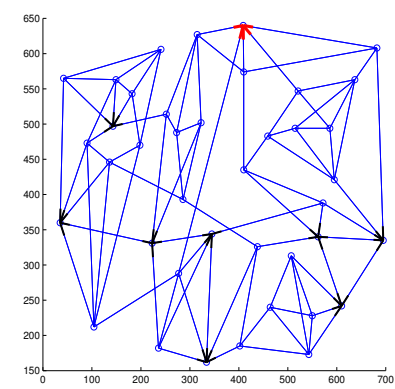

(d) module cost

Figure 22: Worst-case cuts and Nearly Worst-case cuts of the weighted Pioro40 graph by WS

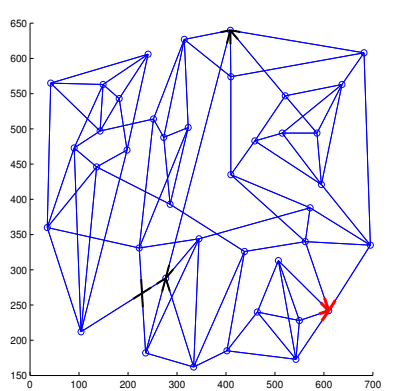

(a) $W_{i, j}$

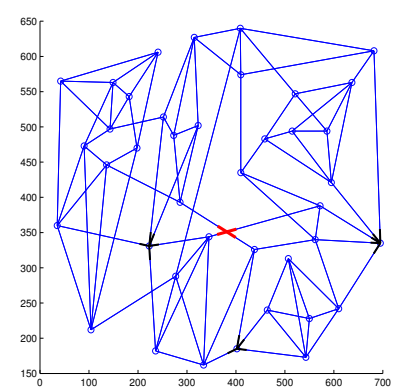

(b) $W_{i, j}^{\star}$

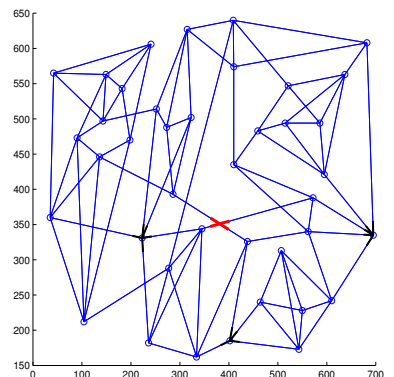

(c) energy consumption

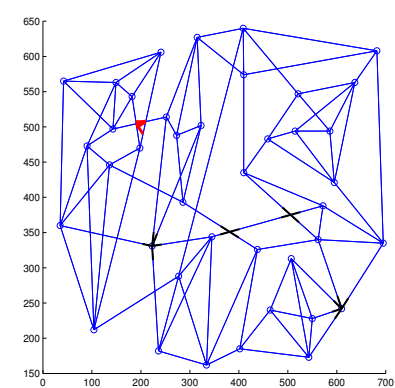

(d) module cost

Figure 23: Worst-case cuts and Nearly Worst-case cuts of the weighted Pioro40 graph by NC

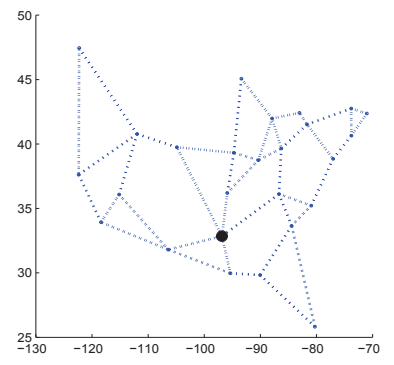

(a) ND

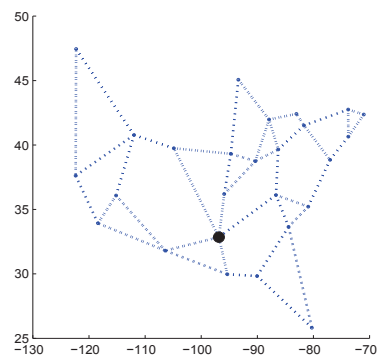

(b) WS

Figure 24: Most vulnerable nodes in an unweighted graph

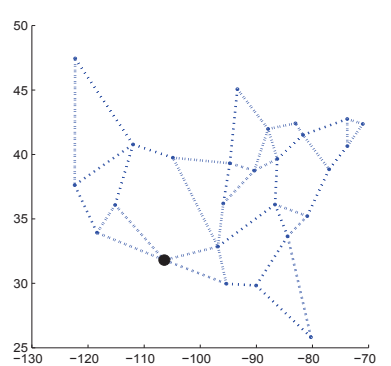

(a) NS

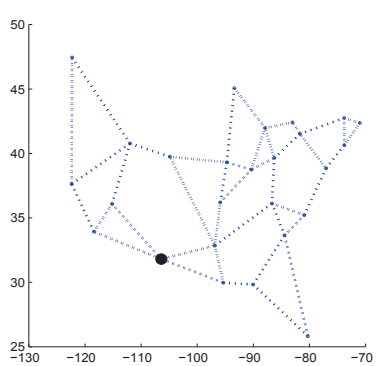

(b) WS

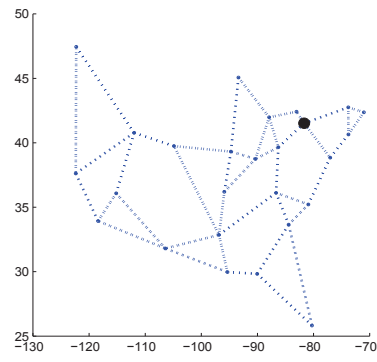

(a) NS

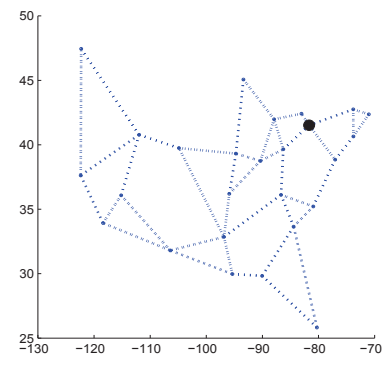

(b) WS

Figure 25: Most vulnerable nodes in a weighted graph based on $W_{i, j}$

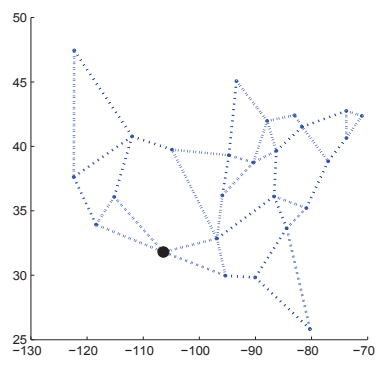

(a) NS

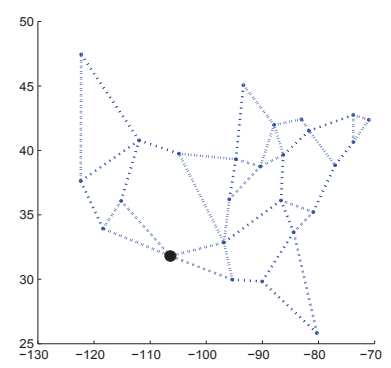

(b) WS

Figure 26: Most vulnerable nodes found in $W_{i, j}^{\star}$ weighted graph

Figure 27: Most vulnerable nodes found in energy consumption weighted graph 


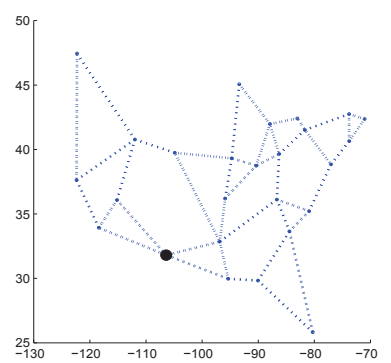

(a) NS

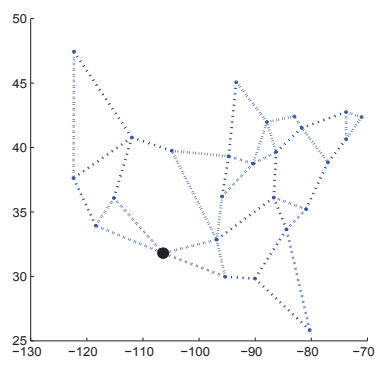

(b) WS

Figure 28: The most vulnerable nodes found by (a) NS (b) WS in a weighted graph based on module cost

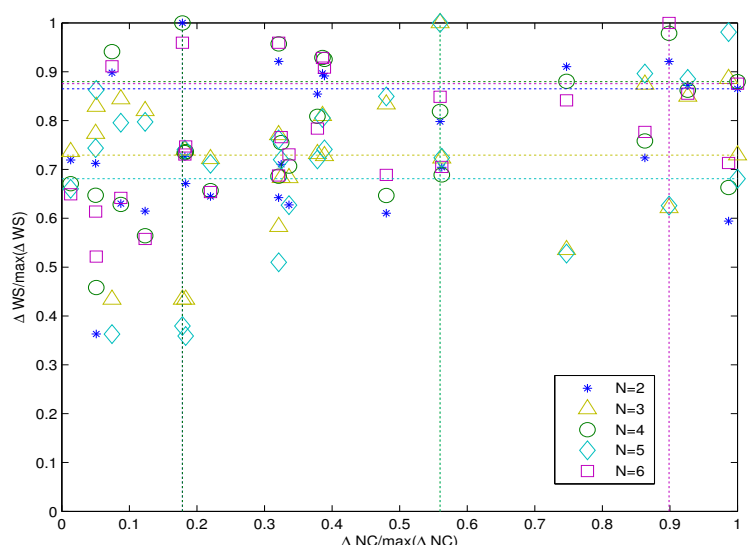

(a) $W_{i, j}$

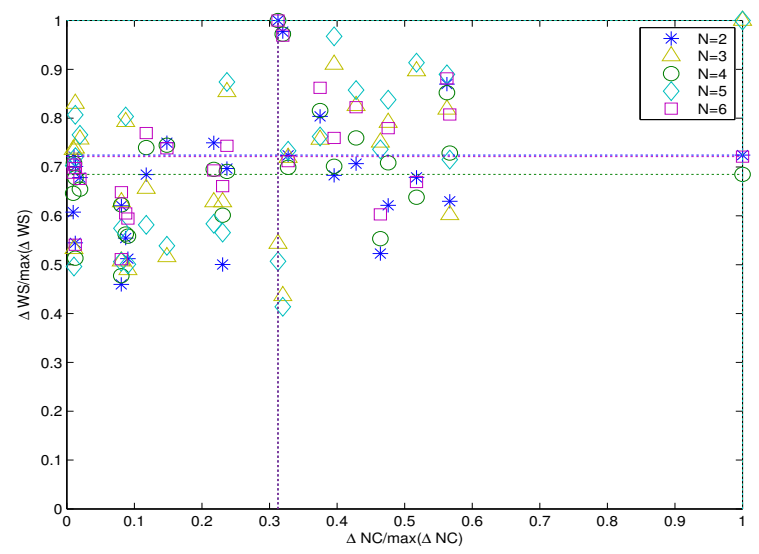

(c) Energy Consumption

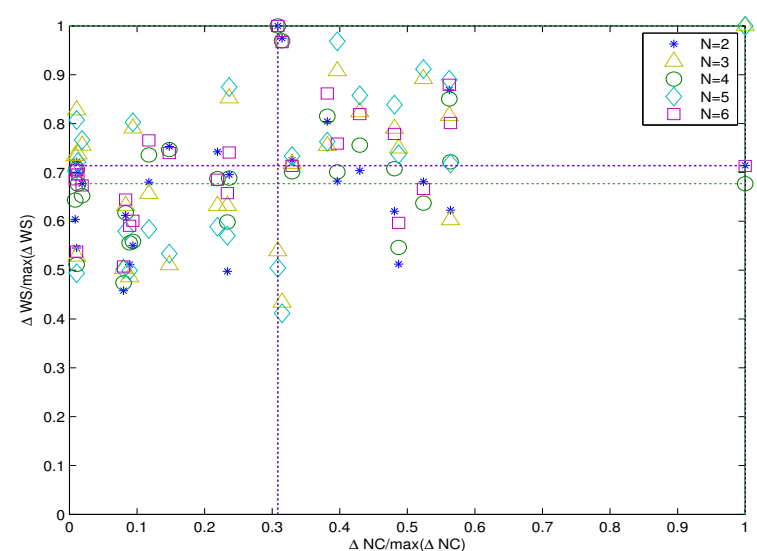

(b) $W_{i, j}^{\star}$

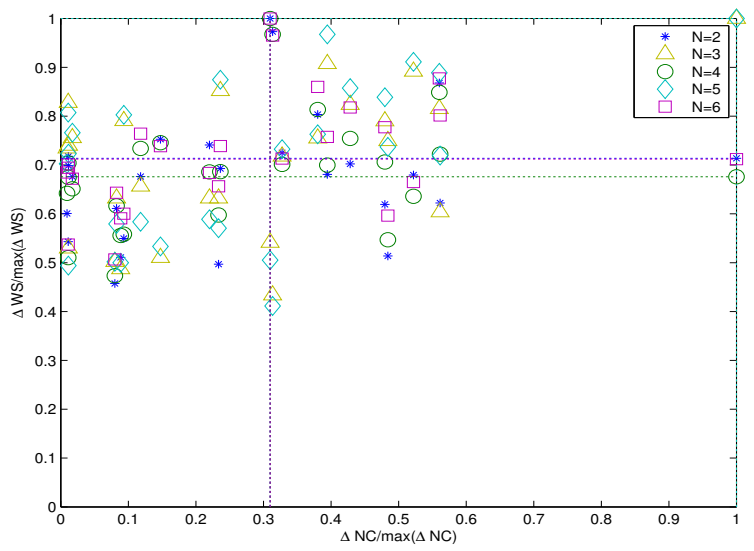

(d) Module Cost

Figure 29: Bi-criteria figures for WS vs. NC with different weighted graphs 


\section{References}

[1] Department of Homeland Security, The National Strategy For the Physical Protection of Critical Infrastructure and Key Assets (Feb. 2003).

[2] T. G. Lewis, Critical Infrastructure Protection in Homeland Security: Defending a Networked Nation, Wiley-Interscience, 2006.

[3] P. Tarvainen, Survey of the survivability of it systems, in: Proc. of 9th Nordic Workshop on Secure IT Systems (NORDSEC'04), Espoo, Finland.

[4] V. Valloppillil, K. W. Ross, Federal standard 1037c: Telecommunications: Glossary of telecommunication terms (1996). URL http: //www.its.bldrdoc.gov/fs-1037/fs-1037c.htm

[5] M. Al-Kuwaiti, N. Kyriakopoulos, S. Hussein, A comparative analysis of network dependability, fault tolerance, reliability, security and survivability, IEEE Communications Surveys \& Tutorials 11 (2) (Second Quarter, 2009) 106-124.

[6] J. S. Foster, Jr., E. Gjelde, W. R. Graham, R. J. Hermann, H. M. Kluepfel, R. L. Lawson, G. K. Soper, L. L. Wood, Jr., J. B. Woodard, Report of the commission to assess the threat to the united states from electromagnetic pulse (emp) attack (2008). URL http: //www . empcommission.org/

[7] M. Fiedler, Algebraic connectivity of graphs, Czechoslovak Mathematical Journal (23(98)) (1973) 298-305

[8] A. Tizghadam, A. Leon-Garcia, On robust traffic engineering in core networks, in: Proc. IEEE Globecom '08, New Orleans, USA.

[9] A. Tizghadam, A. Leon-Garcia, Autonomic traffic engineering for network robustness, IEEE Journal on Selected Areas on Communications 28 (1) (2010) 39-50.

[10] L. C. Freeman, Centrality in social networks: I. conceptual clarification, Social Networks (1) (1978) $215-239$

[11] A. Barrat, M. Barthélémy, R. Pastor-Satorras, A. Vespignani, Architecture of complex weighted networks, Proceedings of the National Academy of Sciences (101(11)) (2004) 3747-3752.

[12] D. Fay, H. Haddahi, A. Thomason, A. Moore, R. Mortier, A. Jamkaovic, S. Uhlig, M. Rio, Weighted spectral distribution for internet topology analysis: Theory and applications, IEEE/ACM Transactions on Networking 18 (1) (2010) 164-176.

[13] S. Neumayer, G. Zussman, R. Cohen, E. Modiano, Assessing the vulnerability of the fiber infrastructure to disasters, in: Proceedings of IEEE Infocom '09, Rio de Janeiro, Brazil, 2009.

[14] A. Jamakovic, S. Uhlig, On the relationship between the algebraic connectivity and graph's robustness to node and link failures, in: Proc. 3rd Conf . Next Gen. Internet Networks (EurNGI) '07, Trondheim, Norway, 2007, pp. 96-102.

[15] W. Liu, K. Pawlikowski, H. Sirisena, Algebraic connectivity metric for spare capacity allocation problem in survivable networks, Computer Communications 34 (12) (2011) 1425-1435.

[16] A. Bigdeli, A. Tizghadam, A. Leon-Garcia, Comparison of network criticality, algebraic connectivity, and other graph metrics, in: Proc. 1st Annual Workshop on Simplifying Complex Network for Practitioners, Venice, Italy, 2009.

[17] S. Neumayer, G. Zussman, R. Cohen, E. Modiano, Assessing the vulnerability of the fiber infrastructure to disasters, IEEE/ACM Transactions on Networking 19 (6) (2011) 1610-1623.

[18] S. Trajanovski, F. Kuipers, P. V. Mieghem, Finding critical regions in a network, in: Proc. of IEEE INFOCOM Fifth International Workshop on Network Science for Communication Networks (NetSciCom 2013), Turin, Italy, 2013

[19] S. Trajanovski, F. Kuipers, P. V. Mieghem, A. Illic, J. Crowcroft, Critical regions and region-disjoint paths in a network, in: Proc. of IFIP Networking 2013, Brooklyn, New York, USA, 2013.

[20] G. Yan, S. Eidenbenz, S. Thulasidan, P. Datta, V. Ramaswamy, Criticality analysis of internet infrastructure, Computer Networks 54 (7) (2010) 1169-1182.

[21] P. Agarwal, A. Efrat, S. Ganjugunte, D. Hay, S. Sankararaman, G. Zussman, The resilience of wdm networks to probabilistic geographical failures, IEEE/ACM Transactions on Networking 21 (5) (2013) 1525-1538.

[22] S. Banerjee, S. Shirazipourazad, P. Ghosh, A. Sen, Beyond connectivity - new metrics to evaluate robustness of networks, in: Proceedings of IEEE 12th International Conference on High Performance Switching and Routing, Cartagena, Spain, 2011, pp. 171-177.

[23] F. R. K. Chung, Spectral Graph Theory, American Mathematical Society, 1997.

[24] C. Godsil, G. Royle, Algebraic Graph Theory, Springer-Verlag, 2001.

[25] S. Rai, D. P. Agrawal, Distributed Computing Network Reliability, IEEE Computer Society Press, 1990

[26] Sndlib http://sndlib.zib.de/home.action.

[27] M. Mezhoudi, C. K. Chu, Integrating optical transport quality, availability, and cost through reliability-based optical network design, Bell Labs Technical Journal 11 (3) (2006) 91-104.

[28] J. Chabarek, J. Sommers, P. Barford, C. Estan, D. Tsiang, S. Wright, Power awareness in network design and routing, in: In Proc. IEEE INFOCOM, 2008. 